\title{
Electrochemical Performance of Silicon/MWCNT Composite Electrodes for Lithium Ion Batteries
}

\author{
T. Cetinkaya*, U. Tocoglu, O. Cenvher, M.O. Guler and H. Akbulut \\ Sakarya University, Engineering Faculty, Department of Metallurgical \& Materials Engineering \\ Esentepe Campus, 54187, Sakarya, Turkey
}

\begin{abstract}
Silicon/MWCNT composite electrodes were produced using high-speed planetary ball milling. Content of MWCNT were altered in silicon/MWCNT composite electrodes and effect of MWCNT content on the electrochemical performance of silicon electrodes were characterized by scanning electron microscopy and X-ray diffraction techniques. Coin type CR2016 test cells were assembled by using produced nanocomposite electrodes. Prepared test cells were electrochemically cycled at a current density of $200 \mathrm{~mA} / \mathrm{g}$. Furthermore, cyclic voltammetry curve of composite electrodes were performed to investigate electrochemical reactions between electrode and electrolyte.
\end{abstract}

DOI: $10.12693 /$ APhysPolA.125.285

PACS: 81.05.Ni, 81.20.Ev, 82.45.Fk, 82.47.Aa

\section{Introduction}

To meet the energy demands of future portable electronics and hybrid electric vehicles (HEVs), advanced electrode materials with high capacities are desired to produce Li-ion batteries (LIBs) with not only high energy density but also high power capability. Silicon is a very promising candidate for anodes because it exhibits an extremely high theoretical capacity of $4200 \mathrm{mAh} \mathrm{g}^{-1}$ and some other advantages such as abundance and non-toxicity [1]. However, severe volume changes (400\%) of $\mathrm{Si}$ anodes during $\mathrm{Li}$ insertion/extraction lead to crack and pulverization, resulting in a rapid capacity fading and limitation of practical applications [2]. Unfortunately, the nanostructured and amorphous forms of silicon have lower electronic conductivity leading to poor charge transport, Coulombic efficiencies, and rate capability characteristics. On the other hand, one-dimensional (1D) structures such as carbon nanotubes (CNTs) are known for their excellent electronic and mechanical properties [3]. Therefore, many studies have been focused on silicon/multiwall carbon nanotubes ( $\mathrm{Si} / \mathrm{MWCNT}$ ) composite electrodes to improve cycleability, conductivity and prevent disintegration of electrodes $[4,5]$.

In the present work, silicon/MWCNT composite electrodes were produced by mechanical alloying of silicon and MWCNTs via high energy mechanical milling method. In order to investigate the effect of MWCNT content on the electrochemical performance of the electrodes, content of MWCNT was altered in the composite structure. It is aimed to contribute the electrochemical performance of the anodes by increasing both conductivity and stress relaxation provided with MWCNTs.

*corresponding author; e-mail: tcetinkaya@sakarya.edu.tr

\section{Experimental details}

MWCNTs (purity 95\%, diameter 50-100 nm) used as reinforcing material for producing $\mathrm{Si} / \mathrm{MWCNT}$ composites, in this study, were supplied from Arry Nano. In order to purification, MWCNTs were stirred in $\mathrm{HNO}_{3}$ for $12 \mathrm{~h}$, and then they were washed with distilled water and dried overnight in an oven. Silicon particles (purity $99.9 \%, 1-20 \mu \mathrm{m}$ in size) were supplied from Alfa Aesar. Three different types of $\mathrm{Si}$ /MWCNT composites were produced with dispersing different amount of MWCNTs $(10,30$, and $50 \mathrm{wt} \%)$. For producing Si/MWCNT composite powders, firstly MWCNTs were homogeneously distributed in $20 \mathrm{ml}$ distilled water by ultrasonic homogenizer for $30 \mathrm{~min}$ to prevent agglomeration of MWCNTs in composite materials. In the second step, silicon powders were added in MWCNT suspension and charged to bowl for mechanical alloying process. For mechanical alloying process, $80 \mathrm{ml}$ stainless steel bowl and $5 \mathrm{~mm}$ balls were used and ball to powder weight ratio was chosen as 10:1. In the last step, silicon powders were mechanically milled in MWCNT suspension to provide mechanical locking of silicon with MWCNT. Finally, obtained suspension was filtered and dried at $80^{\circ} \mathrm{C}$ in vacuum oven for $10 \mathrm{~h}$. By this way, silicon/MWCNT composite powders were produced. Surface morphology and structure of produced $\mathrm{Si} / \mathrm{MWCNT}$ composite powders were characterized by scanning electron microscopy (SEM) and $\mathrm{X}$-ray diffraction (XRD) technique.

For preparation electrodes, 75 wt $\%$ mechanically alloyed active powders and $10 \mathrm{wt} \%$ carbon black mixed with 15 wt\% PVDF (poly(vinylidene fluorides)) binder dissolved in N-methylpyrrolidinone (NMP) solution. The resulting slurry was cast on a copper foil and pasted by doctor blade, then dried at $120^{\circ} \mathrm{C}$ in vacuum oven for $12 \mathrm{~h}$. Coin type CR2016 test cells were assembled in an argon filled glove box. The prepared electrodes were used as a working electrode, Li foil used as counter electrode, and $1 \mathrm{M} \mathrm{LiPF}_{6}$ dissolved in a mixture of ethy- 
lene carbonate (EC) and dimethyl carbonate (DMC) (1:1 in volume) as the electrolyte. The working and counter electrodes were separated with polypropylene (PP) separator. Charge-discharge characteristics were obtained at $298 \mathrm{~K}$ between $50 \mathrm{mV}$ and $1.5 \mathrm{~V}$ at a constant current of $200 \mathrm{~mA} / \mathrm{g}$.

\section{Results and discussion}

Figure 1 shows the surface morphology of unreinforced silicon powders and reinforced silicon powders with different content of MWCNTs. It can be seen in Fig. 1 that when MWCNT content is increased in the composite structures, MWCNTs are distributed homogeneously and create a network structure by covering the silicon particle surfaces, which can be helpful for preventing pulverization of silicon powders during electrochemical cyclic test due to buffering effect of MWCNT. Figure 2 shows XRD patterns of unreinforced silicon and Si/MWCNT composites containing different amount of MWCNT. It is clearly seen in Fig. 2 that when MWCNT content is increased in composite structure, the intensities of main peaks of MWCNT (002) and (101) observed at $2 \theta$ values of $26^{\circ}$ and $44^{\circ}$ increase [6].
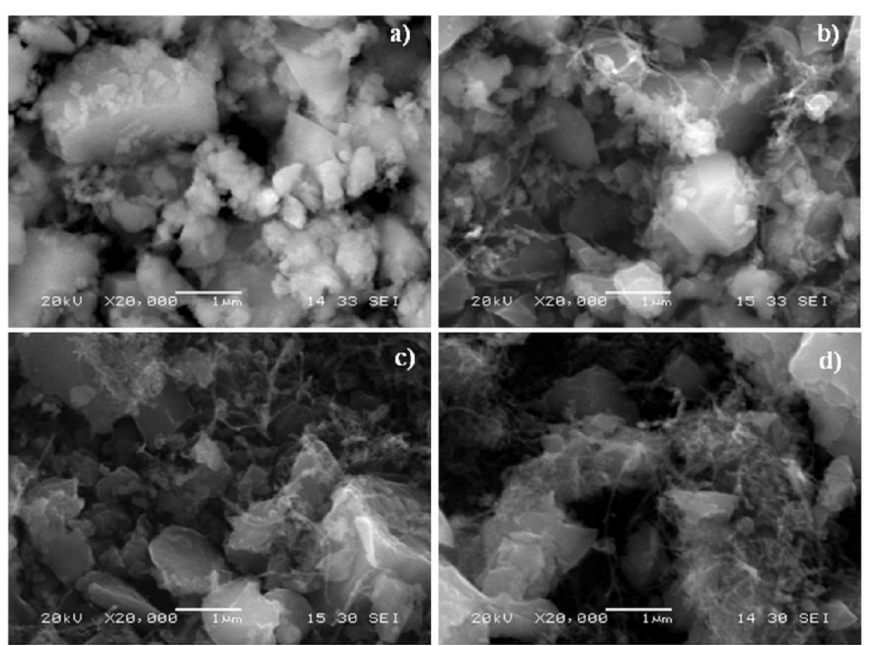

Fig. 1. SEM images of (a) bare $\mathrm{Si}$, (b) $\mathrm{Si}_{0.9}$ $\mathrm{MWCNT}_{0.1}$, (c) $\mathrm{Si}_{0.7} / \mathrm{MWCNT}_{0.3}$, and (d) $\mathrm{Si}_{0.5} /$ $\mathrm{MWCNT}_{0.5}$

The cyclic voltammetry curves of Si/MWCNT composite are shown in Fig. 3a. The cyclic voltammetry curves of Si/MWCNT composite electrode clearly showed peaks corresponding to insertion and extraction of $\mathrm{Li}$ ions. In the cathodic part of $\mathrm{CV}$ curves of Si/MWCNT composites, the peak between $0.6 \mathrm{~V}$ and $0.8 \mathrm{~V}$ appearing during the first cycles of $\mathrm{CV}$ curves are due presumably to an irreversible reaction for the solid electrolyte interphase (SEI) formation in the Li insertion into the Si/MWCNT composites.

It is known that SEI film is an insulator for electrons and a good conductor for lithium ions, which can freely insert and de-insert through this passive film.

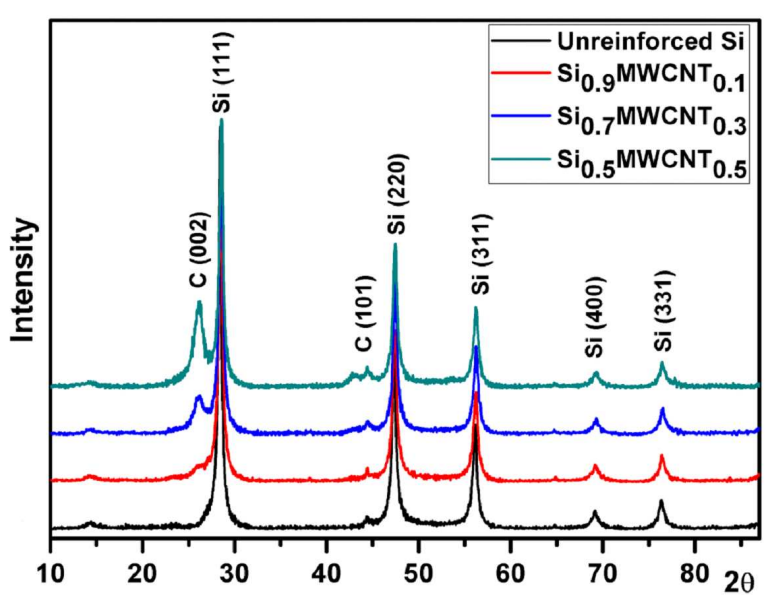

Fig. 2. XRD patterns of unreinforced silicon, $\mathrm{Si}_{0.9} \mathrm{MWCNT}_{0.1}, \mathrm{Si}_{0.7} \mathrm{MWCNT}_{0.3}$ and $\mathrm{Si}_{0.5} \mathrm{MWCNT}_{0.5}$ composite powders.
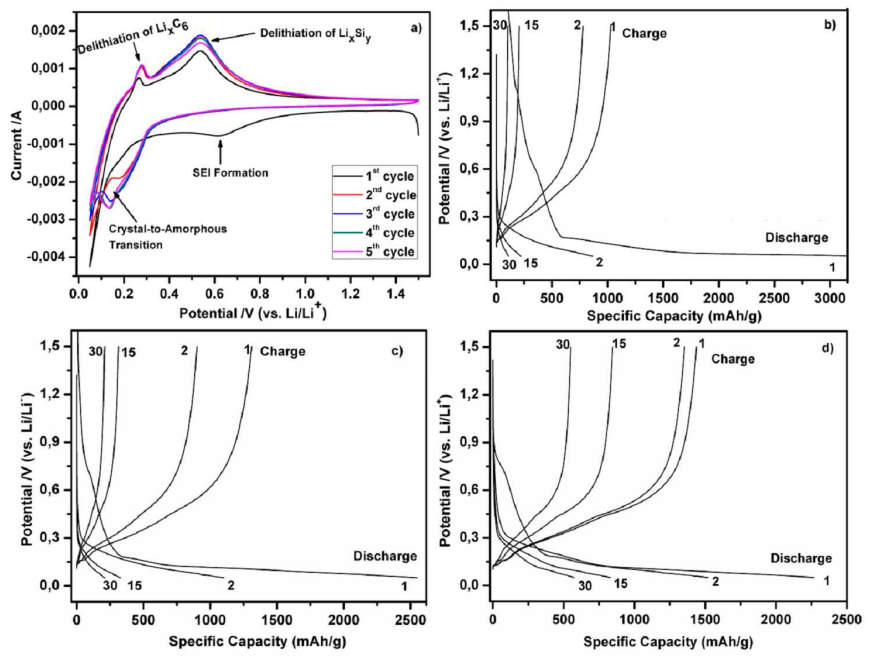

Fig. 3. (a) Cyclic voltammetry curves of produced $\mathrm{Si} / \mathrm{MWCNT}$ composite electrode, (b), (c), and (d) charge-discharge profile of $\mathrm{Si}_{0.9} \mathrm{MWCNT}_{0.1}$, $\mathrm{Si}_{0.7} \mathrm{MWCNT}_{0.3}$, and $\mathrm{Si}_{0.5} \mathrm{MWCNT}_{0.5}$, respectively.

During the second cycle, the peak at $0.15 \mathrm{~V}$, which is absent at the first cycle, indicates the crystal-to-amorphous transition of silicon. In the anodic part of the $\mathrm{CV}$ curves of $\mathrm{Si} / \mathrm{MWCNT}$ composite electrodes, the peak around $0.2 \mathrm{~V}$ shows that lithium extraction between graphene layers of MWCNT and de-alloying reaction of silicon with lithium was observed between $0.3 \mathrm{~V}$ and $0.6 \mathrm{~V}[7]$.

Figure $3 \mathrm{~b}-\mathrm{d}$ demonstrates galvanostatic charge and discharge profile of $\mathrm{Si} / \mathrm{MWCNT}$ composite electrodes in the coin cells at a current density of $200 \mathrm{~mA} / \mathrm{g}$ between $0.05 \mathrm{~V}$ and $1.5 \mathrm{~V}$ vs. $\mathrm{Li} / \mathrm{Li}^{+}$. It is clearly seen from the charge-discharge profile curves that the plateaus at $0.6 \mathrm{~V}$ and $0.2 \mathrm{~V}$ during discharge, which show SEI formation and lithiation of silicon and the plateaus at $0.4 \mathrm{~V}$, which show delithiation of silicon with lithium clearly overlapped with the cyclic voltammetry curves [8]. 
Furthermore, as can be seen in Fig. 3a-d, when MWCNT content is increased in composite structure, the capacity retention of composite increases.

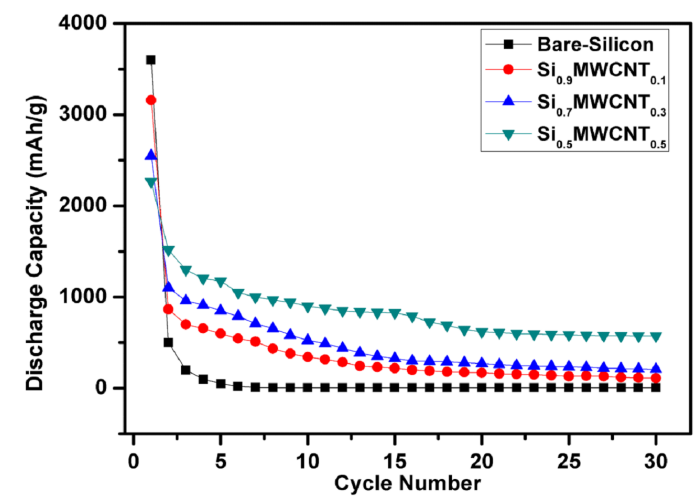

Fig. 4. Discharge capacity vs. cycle number of $\mathrm{Si} / \mathrm{MWCNT}$ composite electrodes.

Cycling performance of unreinforced silicon electrode and produced $\mathrm{Si} / \mathrm{MWCNT}$ composite electrodes at a constant current density $200 \mathrm{~mA} / \mathrm{g}$ are shown in Fig. 4. It is seen from Fig. 4 that unreinforced silicon electrode loses almost all capacity after few cycles. However, $\mathrm{Si} / \mathrm{MWCNT}$ composite electrodes demonstrates better irreversible capacity and when MWCNT content is increased in composite structures, the composite electrodes show higher stable capacity and among the electrodes, $\mathrm{Si}_{0.5} / \mathrm{MWCNT}_{0.5}$ composite electrode shows the best capacity retention and stable capacity and this composite indicates $570 \mathrm{mAh} / \mathrm{g}$ discharge capacity even after 30 cycles.

\section{Conclusion}

Si/MWCNT composite electrodes were successfully produced by high speed planetary ball milling. When
CNT content is increased in the composite structure, MWCNTs are distributed homogeneously and create a conductive network caused improving cycleability, discharge capacity and prevents pulverization of the electrodes. The best performance among the nanocomposite electrodes obtained from the $\mathrm{Si}_{0.5} / \mathrm{MWCNT}_{0.5}$ composite electrode, which indicated $550 \mathrm{mAh} / \mathrm{g}$ discharge capacity even after 30 cycles.

\section{Acknowledgments}

This work is supported by the Scientific and Technological Research Council of Turkey (TÜBİTAK) under the contract number 111M021. The authors thank the TÜBİTAK MAG workers for their financial support.

\section{References}

[1] Y. Fan, Q. Zhang, C. Lu, Q. Xiao, X. Wang, B. Tay, Nanoscale 5, 1503 (2013).

[2] K.S. Park, K.M. Min, S.D. Seo, G.H. Lee, H.W. Shim, D.W. Kim, Mater. Res. Bull. 48, 1732 (2013).

[3] R. Epura, M.K. Dattab, P.N. Kumta, Electrochim. Acta 85, 680 (2012).

[4] A. Gohier, B. Laïk, K.H. Kim, J.L. Maurice, J.P.P. Ramos, C.S. Cojocaru, P.T. Van, Adv. Mater. 24, 2592 (2012).

[5] W. Wang, R. Epur, P.N. Kumta, Electrochem. Commun. 13, 429 (2011).

[6] L. Yue, H. Zhong, L. Zhang, Electrochim. Acta 76, 326 (2012).

[7] T. Cetinkaya, M.O. Guler, H. Akbulut, Microelectron. Eng. 108, 169 (2013).

[8] Y. Zhang, X.G. Zhang, H.L. Zhang, Z.G. Zhao, F. Li, C. Liu, H.M. Cheng, Electrochim. Acta 51, 4994 (2006). 